\title{
Schädigung des Lichtsinnes bei den Myopen.
}

Mit Zugrundelegung eines in Gruppe $\mathrm{F}$

des I. internationalen schulhygienischen Kongresses

in Nürnberg am 8. April 1904 gehaltenen Vortrages.

Von

Generalarzt Dr. Seggel

in München.

Mit 5 Figuren im Text.

Auf Schädigung der Sehschärfe bei den Kurzsichtigen habe ich wohl als erster und zwar schon im Jahre $1878^{1}$ ) und dann in einer eingehenderen Arbeit ${ }^{2}$ ) 1884 hingewiesen.

Angeregt durch die Mitteilung Samelsohns ${ }^{3}$, dass sich bei Refraktionsanomalien - besonders bei der Myopie - der Lichtsinn herabgesetzt finde, habe ich dann eingehendere Untersuchungen mit der Wolffbergschen zentralen Farbensinnprobe gemacht und hierbei den Ausspruch Samelsohns bestätigt gefunden. Auf Grund dieser Untersuchungen stellte ich in einer folgenden Arbeit ${ }^{4}$ ) den Satz auf, dass Abnahme des zentralen Farbenmaximums für Blau und Rot ein Anzeichen, und zwar oft das einzige, für das Bestehen des myopischen Prozesses, der mit Bulbusverlängerung einhergeht, sei. Da die Prüfung des quantitativen Farbensinnes gewissermassen auch eine Prüfung des Lichtsinnes darstellt - es wird ja die Wahrnehmung farbigen Lichtes dabei geprüt -, so schloss ich, dass mit Abnahme der zentralen Farbenwahrnehmung i. e. des $c F m$ auch eine Abnahme

1) Die Zunahme der Kurzsichtigkeit in den höhern Unterrichtsanstalten. Bayer. arztl. Intelligenzbl. 1878.

2) Über normale Sehschärfe und die Beziehungen der Sehschärfe zur Refraktion. v. Graefe's Areh. f. Ophtalm. Bd. XXX. 2. 1884.

3) Compte rendu de travaux du congrès internationale périodique de sciences médicales, 8 we Session. Copenhague 1 884 . Tom. III. p. 6 .

4) Seggel, Über die Prüfung des Licht- und quantitativen Farbensinnes usw. Arch. f. Augenheilk. Bd. XVIII. S. 303. 
des Lichtsinnes signalisiert und dass bei Myopie neben Abnahme der Sehschärfe in Herabsetzung des Lichtsinnes auch ohne ophthalmoskopisch nachweisbare Veränderungen die Zeichen des durch Dehnung der innern Augenhäute bewirkten sog. myopischen Prozesses zu erkennen seien.

Meine Anschaumg hat wohl Zustimmung gefunden, wurde aber nicht weiter verfolgt, hauptsächlich wohl deshalb, weil die angewandte Methode nicht genuigend beweiskräftig erschien. Ich habe deshalb, nachdom ich durch dienstliche Verpflichtungen nicht mehr abgehalten bin, die Sache weiter verfolgt und an zwei hiesigen Instituten, einem männlichen und einem weiblichen, den Lichtsinn nach andern einwandsfreieren Methoden untersucht und zwar auf dreierlei Art. Die Hilfsmittel, die ich dazu benutate, waren meine Lichtsinntafeln, die sich mir inzwischen als zuverlässig erwiesen hatten, und das Förstersche Photometer. Mit den erstern ${ }^{1}$ ) prüfe ich die Unterschiedsempfindlichkeit $L d$ (Sensus Luminis discernendi) i. e. den kleinsten noch wahruehmbaren Unterschied zwischen zwei objektiven Hellig. keiten, mit dem letztern die Reizempfindlichkeit $L i$ (Sensus $L$. irritationis) d. i. die geringste noch wahrnehmbare Helligkeit. Bei meinen Lichtsinntafeln bildet auf Tafel I der weisse, auf den drei andern Tafeln grauer Grund in verschiedener Abstufung mit den nach Snellenschem Prinzip entworfenen schwarzen Buchstaben den Kontrast. Die Prüfung wurde dann auch in analoger Weise wie bei der Sehschärfeprüfung nach Korrektion etwaig vorhandener Ametropie, also auch der Kurzsichtigkeit, und selbst der minimalsten Grade und zwar in etwas abgekiurzten Verfahren in der Weise vorgenommen, dass in der Entfernung, in welcher Snellen 6 auf der weissen Tafel gelesen wird, festgestellt wurde, welche Reihe der vierten - dunkelsten - Tafel noch gelesen werden kann. Da ein Auge mit normaler Unterschiedsemptindlichkeit auf jene Entfernung Nr. 18, d, i. die vierte Reihe noch zu lesen vermag, so bildet 18 den Zähler und die Reihe, welche von dem Jntersuchten noch gelesen wird, den Nenner des den Lichtsinn ausdrückenden Bruches. Erkennt z. B. jemand Snellen 6 auf Tafel I auf $4 \mathrm{~m}$, so wird in dieser Entfernung geprüft, welche Buchstabenreihe der Tafel IV noch erkannt werden kann. Kann nur die dritte Reibe i. e. Snellen 24 erkannt werden, ausgedriickt durch ${ }^{4 / 24}$, so ist $L d{ }^{18 / 24}$. Wird

1) Seggel, Sehprobentafeln zur Prüfung des Lichtsinnes. München, Lit. artist. Anstalt fon Theodor Riedel, nun Fr. Bassermann. 
Snellen 6 auf Tafel $\mathrm{I}$ auf $6 \mathrm{~m}$ und in der gleichen Entfernung Snellen 12 auf Tafel IV erkannt, ausgedrückt durch $6 / 12$, so ist Ld $18 / 12,{ }^{18 / 18}$ bedeutet also normale, ${ }^{18 / 24}$ unternormale und $18 / 12$ bessere Unterschiedsempfindlichkeit als die normale. Werden nicht sämtliche Buchstaben einer Reihe gelesen, so wird die $Z$ wischenzahl als Nenner z. B. $20=<18,22>24$, angenommen. In den beigegebenen Tabellen ist der Zähler 18 weggelassen und nur der Nenner mit halbfetten Ziffern angeführt. Um eine Übereinstimmung mit den beiden andern Untersuchungsarten herbeizuführen, wurden die gefundenen Werte in 72 tel umgewandelt und mit kleinen Ziffern dargestellt, in vorstehenden Beispielen also ${ }^{18} / 24={ }^{54} / 72$ : ${ }^{18} / 12={ }^{108} / 72,{ }^{18} / 20=65 / 72$.

Die Reizempfindlichkeit $L i$ wurde zunächst mit dem Försterschen Photometer in bekannter Weise geprüft, wobei allerdings nur die Reizempfindlichkeit des Auges im allgemeinen bestimmt wird. Um nun auch die zentrale Reizempfindlichkeit zu bestimmen, habe ich nach der Angabe Treitels ${ }^{1}$ ) anstatt der schwarzen Striche auf weissem Grunde im Hintergrunde des Photometerkastens ein kleines weisses Quadrat von $3 \mathrm{~mm}$ Seite auf einer mit schwarzem Sammet bezogenen Tafel angebracht und nun bestimmt, bei welchem Durchmesser des Diaphragma das weisse Quadrat noch als belle Stelle gesehen werden kann. Bei normalem zentralen Lichtsinn ist zur Wahrnehmung des weissen Quadrates noch ein Durchmesser des Diaphragma von $3 \mathrm{~mm}$ notwendig, während bei der gewöhnlichen Försterschen Methode ein Durchmesser von $2 \mathrm{~mm}$ normalen Lichtsinn angibt. Die Formel für Berechnung des Lichtsinnes verwandelt sich daher bei der Treitelschen Methode in $H={ }_{1 / s} a^{2}$ und $L=\frac{1}{1 / 3 H}$. Zur Unterscheidung beider Methoden zur Prüfung der Reizempfindlichkeit babe ich für die Förstersche Methode die Bezeichnung Lia, für die Treitelsche: Lic gewählt (Sensus Luminis irritationis centralis).

In den Tabellen geben in den Rubriken für die Reizempfindlichkeit Lia und Lic die fettgedruckten Zahlen den bei der Prüfung gefundenen kleinsten Durchmesser des Diaphragma an. Um eineu gleichmässigen Überblick zu gewähren, sind auch sämtliche Werte für $L i$ ebenso wie für $L d$ Unterschiedsempfindlichkeit auf den Generalnenner von 72 gebracht, welcher sich mir als der praktischste er-

1) Treitel, Über Hemeralopie u. Untersuchung des Lichtsinnes. v. Gra ef e's Arch. f. Ophthalm. Bd. XXXI, 1. S. 259. 
wies, z. B. Lia $2 \mathrm{~mm}={ }^{72 / 72}$, Lia $3 \mathrm{~mm}=36 / 72, \quad L i c 3 \mathrm{~mm}=72 / 725$ Lie $4 \mathrm{~mm}=3 / 5=41 / 72$.

Es könnte mir entgegengehalten werden, dass auch nach der. von mir gewählten vereinfachten Treitelschen Methode nicht, wenigstens nicht ausnahmslos, die zentralo Reizempfindlichkeit gemessen wird, sondern unter besondern Umständen die circumforeale, $d$. der $t i$ des die zentrale Netzhautgrabe umgebenden Stäbchenkranzes; da die Fovea selbst keine - für das Licht empfindlichere - Stäbchen, sondern nur Zapfen enthält. Um diese mögliche Fehlerquelle auszuschliessen, hätte ich eines komplizierteren Apparates und vor allem sehr exakt beobachtender Untersuchungspersonen bedurft, wie es mir wenigstens die jüngern $Z$ Zöglinge nicht boten. Gleichwohl glaube ich mit meinen drei einfachen Methoden, wenn ich mit denselben auch nicht den strengen Forderungen einer exakt wissenschaftlichen Prüfung nachgekommen bin, doch den Nachweis geminderten Lichtsinnes bezw. von Hemeralopie bei Myopie sicher erbringen zu können.

Nachfolgende Ausfuhrungen bilden zugleich die Ergänzung zu zwei vorausgegangenen Arbeiten $\left.{ }^{1}\right)^{2}$ ), auf die ich auch hinsichtich des Untersuchungsmaterials verweisen kann. Hinsichtlich des letatern habe ich nur zu bemerken, dass dasselbe hier nur einen Jahrgang, im ganzen 281 Zöglinge, nicht wie in den frubern Arbeiten 20 Jahrgänge urnfasst. Ferner ist anzufugen, dass an den beiden Instituten - Internaten -, an denen ich meine Untersuchungen machte, die hygienischen Einrichtungen fast allen Anspritchen entsprechen, insbesondere was die Beleuchtung betrift. Grosse helle, meist nach Norden gelegene Arbeitssäle und eine reichliche künstliche Beleuchtung - so genannte gemischte mittels Auer-bezw. Starklicht und Milchglasschirmen, wobei die Lichtintensität mindestens 25 Meterkemenstärke in Weiss gemessen an jedem Platze beträgt, sind rorhanden. Etwas ungünstiger gestalten sich die Verhältnisse für die männlichen Zöglinge dadurch, dass dieselben überwiegend mit hereditärer Anlage zur $\mathbf{M y -}$ opie belastet sind, und dass dieselben das öfentliche benachbarte Gymnasium besuchen, dessen Tagesbeleuchtungsverhältnisse wenigstens in Winter an den fruhen Morgen- und späten Nachmittagsstunden zu wänschen ubrig lassen. Die Arbeitsceit in beiden Instituten ist im Verhältnis zu den. Stunden körperlicher Übungen eine

1) Üher den Einfluss der Beleuchtung auf die Sehschärfe und die Entstehung der Kurzsichtiglreit, Münch. med. Wochenschr. 1897, Nr. 37 u. 38.

2) Neine Erfahrungen über Eintritt und Fortschreiten der Myopie usw. v. Graefe's Arch. f. Ophthalm. Bd. LVI. 3. 
grosse, auch Druck und Papier lassen noch zu wünschen übrig. Die männlichen Zöglinge standen im Alter von 10 bis 19, die weiblichen im Alter von 8 bis $16 \frac{1}{2}$ Jahren. Durch das jüngere Alter sind letztere ebenfalls etwas günstiger gestellt, als die männlichen Zöglinge. Hinsichtlich des Vorganges bei der Prüfung ist noch zu bemerken, dass die Untersuchungen auf Sehschärfe und $L d$ nach genauester optometrischer und ophthalmoskopischer Refraktionsbestimmung nur bei gutem Tageslicht, die Lichtsinnprüfungen mit dem Försterschen Photometer immer erst nach einer Dunkeladaption von wenigstens 20 Minuten gemacht wurden.

Da der Nachweis für den schädlichen Einfluss der Myopie auf das Auge überzeugend nur auf statistischem Wege geführt werden kann, war es notwendig, eine Reihe von Tabellen und Diagrammen herzustellen, die der nachfolgenden Darstellung zu Grunde liegen.

Zunächst zeigt Tabelle $I$, wie sich Sehschärfe und Lichtsinn und zwar letzterer nach drei Richtungen als $L d$, Lia und $L i c$ bei beiden Geschlechtern im allgemeinen verhalten.

Tabelle I. Sebschärfe und Lichtsinn nach Geschlechtern.

\begin{tabular}{|c|c|c|c|c|c|c|c|}
\hline \multirow{3}{*}{ Gesehleeht } & \multirow{3}{*}{$\begin{array}{c}\text { ZahI } \\
\text { der } \\
\text { Unter- } \\
\text { suchten }\end{array}$} & \multirow{3}{*}{$\begin{array}{c}\text { Dureh- } \\
\text { sehnitt- } \\
\text { icher } \\
\text { Myopiegrad }\end{array}$} & \multirow{3}{*}{$\begin{array}{c}\text { Prozent- } \\
\text { satz } \\
\text { dar } \\
\text { Myopen }\end{array}$} & \multirow{3}{*}{$\begin{array}{l}\text { Dureh- } \\
\text { sehnitz- } \\
\text { liche } \\
\text { Sehschärfe }\end{array}$} & \multicolumn{3}{|c|}{ Durchschnittlicher Lichtsinn } \\
\hline & & & & & \multirow{2}{*}{$\begin{array}{c}\text { Unter- } \\
\text { sehieds- } \\
\text { ompfindlich- } \\
\text { keit } L /\end{array}$} & \multicolumn{2}{|c|}{ Reizempfindhehkeit $L$} \\
\hline & & & & & & $\begin{array}{c}\text { nach } \\
\text { Forster } a\end{array}$ & $\begin{array}{c}\text { nach } \\
\text { Treitel }\end{array}$ \\
\hline Weiblich & 160 & 2,0 & 18 & $87 / 78$ & $14,8 \quad 90 / 72$ & $2,1 \quad 68 / 72$ & $3,6 \quad 50 / 79$ \\
\hline Männlich & 121 & 2,9 & 39 & $72 / 72$ & $20,7 \quad 61 / 72$ & $2,42^{58 / 72}$ & $5,7 \quad 21 / \pi$ \\
\hline Zusammen & 281 & 2,56 & 28,5 & $80 / 72$ & $17,2 \quad 54 / 72$ & $2,2465 / 72$ & $4,44^{35} / 70$ \\
\hline
\end{tabular}

Diese Tabelle bedarf keiner näheren Erklärung. Wir ersehen aus derselben mit einem Blick, dass Sehschärfe und Lichtsinn nahezu in gleichem Verhältnis bei den etwas besser situierten Mädchen besser sind als bei den Knaben. Erstens ist die Durchschnittssehschärfe, welche bei den Mädchen eine erheblich bessere ist, als die normale, nämlich ${ }^{87} / 72$, bei den Jünglingen gerade noch normal $=\pi / 72$, und zweitens sinkt bei den Jünglingen der Durchschnittslichtsinn und zwar in allen drei Modalitäten unter die Norm, bei $L i$ sogar sehr beträchtlich - Ld = ${ }^{61 / 72}$, Lia ${ }^{56 / 72}$, Lic nur ${ }^{21 / 72}$-, während dies bei den Mädchen eigentlich nur bei der zentralen Reizempfindlichkeit der Fall ist, da $L i c={ }^{50} /{ }_{12}$. Schon die dritte und vierte Kolumne der Tabelle I, welche den durchschnittlichen Myopiegrad 
und den Prozentsatz der Myopen angeben, deuten darauf hin, dass der böhere Myopiegrad (2,9 gegen 2,0) und der höhere Myopienprozentsatz (39 gegen 18) die Ursache der geringeren Sehschärfe und des noch schlechteren Lichtsinnes bei den Knaben und Jünglingen sein wird.

Als genauerer Nachweis hierfür dient Tabelle II. Diese Tabelle lässt ersehen, wie sich Sehschärfe und Lichtsinn bei den verschiedenen Refraktionszuständen verhalten. Demnach ist bei Myopie und myopischem Astigmatismus der Lichtsinn am schlechtesten. Ja bei reiner Myopie ist trotz besserer Durchschnittssebschärfe $\left({ }^{69 / 72}\right)$ der Lichtsinn mit ${ }^{63 / 72},{ }^{45 / 72}$ und ${ }^{18 / 72}$ schlechter als bei hypermetropischem Astigmatismus (S $6 / 2$, $L d$ ${ }^{81 / 72}$, Lia ${ }^{68 / 72}$, Lic ${ }^{38 / 72}$ ) und höhergradiger Hypermetropie (S ${ }^{66} / 72, L d{ }^{77} / 72$, tia ${ }^{50 / 72}$, Lic ${ }^{24 / 72}$ ), darin liegt doch sicher der Beweis, dass der myopische Prozess den Lichtsinn schädigt. Wenn bei myopischem Astigmatismus der Lichtsinn noch schlechter ist als bei reiner Myopie, so erklärt sich dies dadurch, dass es sich fast durchweg um zusammengesetzten Astigmatismus handelt, und dass Augen mit solcher Komplikation wegen ihrer schlechten Sehschärfe (53/, durchschnittlich) noch stärker bei der Naharbeit angestrengt werden als die rein myopischen Angen.

TabelleII. Sehschärfe und Lichtsinn abhängig von der Refraktion.

\begin{tabular}{|c|c|c|c|c|c|c|c|c|}
\hline \multirow{3}{*}{ Mefraktion } & \multirow{3}{*}{$\begin{array}{l}\text { Prozent- } \\
\text { satz }\end{array}$} & \multirow{3}{*}{ Sehsobărfo } & \multicolumn{6}{|c|}{ Durchschnittlicher Lichtsinn } \\
\hline & & & \multirow{2}{*}{\multicolumn{2}{|c|}{$\begin{array}{l}\text { Untersehieds- } \\
\text { empfindliuhkeit } \\
\text { Ld }\end{array}$}} & \multicolumn{4}{|c|}{ Reizempefndlioh keit $L i$} \\
\hline & & & & & $\begin{array}{l}n \\
\text { Fors } \\
\text { For }\end{array}$ & & $\operatorname{Tr} \theta^{n}$ & $\begin{array}{ll}\mathrm{h} \\
\theta 1 c\end{array}$ \\
\hline $\left.\begin{array}{c}\text { Emmetropie } \\
\text { bei Mädchen }\end{array}\right\}$ & 70,3 & $98 / 73$ & 14,1 & $82 / 72$ & 1,873 & $84 / 82$ & 3,08 & $2 \% / 32$ \\
\hline$\underset{\text { Emberbaupt }}{\text { Emmetropie }}\}$ & 59,5 & $30 / 78$ & 15,5 & $85 / 78$ & 1,9 & $80 / 72$ & 3,46 & $54 / 72$ \\
\hline $\left.\begin{array}{c}\text { Astigmatismus } \\
\text { hypermetropicus }\end{array}\right\}$ & 5,0 & $6 \% / r z$ & 16 & $81 / 72$ & 2,14 & $68 / 72$ & 4,23 & $38 / 72$ \\
\hline $\begin{array}{c}\text { Hochgradige } \\
\text { Hypexmetropie }\end{array}$ & 3,5 & $66 / 72$ & 16,6 & $77 / 72$ & 2,675 & $50 / \pi 2$ & $\tilde{b}, \mathbf{f}$ & $24 / 7 \pm$ \\
\hline Myopie & 28,5 & $69 / 78$ & 20 & $65 / 72$ & 2,8 & $45 / 72$ & 6,25 & $18 \%$ \\
\hline $\left.\begin{array}{c}\text { Astigmatismua } \\
\text { myopiens }\end{array}\right\}$ & 3,2 & $58 / 72$ & 32 & $40 / 72$ & 3,3 & $2 \pi / 72$ & 6,75 & $18 / 72$ \\
\hline
\end{tabular}

Übersichtlicher noch als durch Tabelle II wird das Verhalten des Lichtsinnes bei den verschiedenen Refraktionszuständen durch Diagramm.1 dargestellt. In dieser Zeichnung stellt die einfach gestrichelte schwarze Linie den Wert der Sehschärfe (S), die ausgezogene schwarze 
Linie die Unterschiedsempindlichkeit $L d$, die gestrichelt punktierte Linie die Reizempfindlichkeit nach Förster Lia, die ausgezogene Doppellinie die zentrale Reizempfindlichkeit nach Treitel Lic dar und wir sehen nun mit einem Blick, wie die drei ausgezogenen Limien, welche den Lichtsinn darstellen, von der Emmetropie bei den weiblichen Personen, bei welchen sowohl Sehschärfe als Lichtsinn besser ist als bei den männlichen ${ }^{1}$ ), zur Myopie bezw. myopischem Astigmatismus rasch abfallen, und zwar $\mathbf{S}$ von $93, L d$ von 92, Lia von 84, Lie von 72 auf $53,40,27$ und sogar 16 Zweiundsiebzigstel.

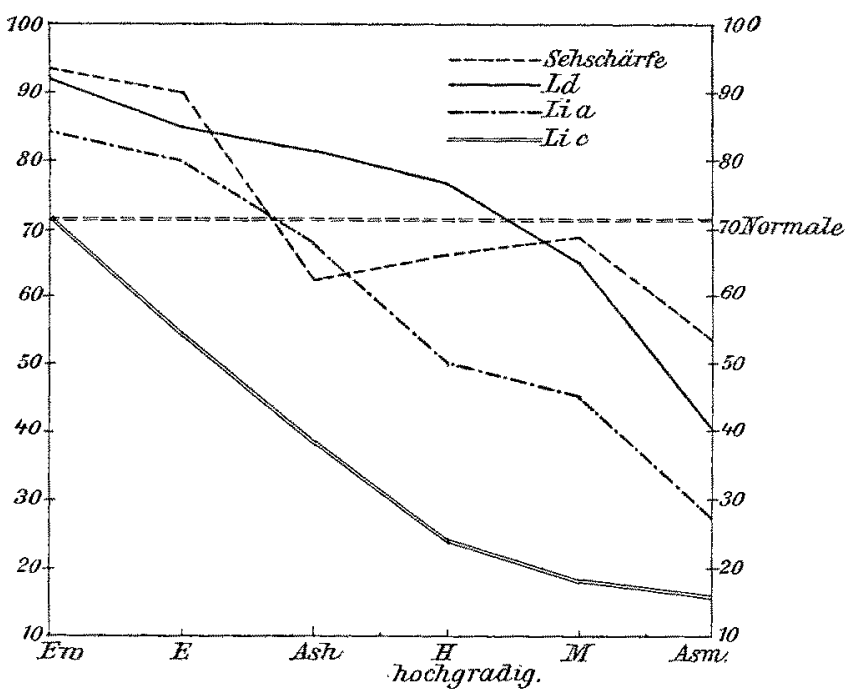

Fig. 告,

Besonders möchte ich bei Diagramm 1 noch darauf hinweisen, wie die drei Modalitäten des Lichtsinnes zwar in drei verschiedenen Abstufungen, aber doch sonst ganz gleichmässig - nahezu parallel abfallen und bei Myopie bezw. myopischem Astigmatismus ihre tiefste Stufe einnehmen. Die einfach gestrichelte Kurve, welche die Sehschärfe dar-

1) Der von mir fräher ausgesprochene Satz 2), dass bei Knaben der myopische Prozess die Sehschärfe viel weniger beeinträchtigt als bei den Mädchen, wird dadurch nicht berührt, denn nur bei den myopischen Mädchen ist die Sehschärfe geschädigt, die Zahl der Myopen tritt aber bei den Mädchen gegen die bei den Knaben weit zurück.

2) Arch. f. Augenheilk. Bd. XVIIT. S. 317. 
stellt, hat dagegen einen andern nicht gleichmässig abfallenden Veriauf, sie erhebt sich sogar wieder gegen die Myopie zu, weil eben bei dieser die Sehschärfe weniger geschädigt wird, als der Lichtsinn; bei hypermetropischem Astigmatismus und höhergradiger Hypermetropie ist gegenteilig der Lichtsinn wenigstens $L d$ und Li $n$, besser als die Sehschärfe; die Sehschärfe ist eben bei diesen beiden letztern Refraktionszuständen nicht durch besonders einwirkende Schädlichkeiten verschlechtert worden, sondern ist als angeborener Zustand an und für sich schlechter. Das höhergradig hypermetropische Auge ist ja bekanntlich ein in der Entwicklung mehr oder weniger zurückgebliebenes Auge. Das kurzsichtige Auge war aber abgesehen von den angeborenen hochgradigen Formen ursprünglich ein gutes und hat erst später an Sehschärfe und Lichtsinn eingebüsst - Im Diagramm 1 sehen wir bei Ash die gestrichelt-punktierte und einfache ausgezogene Linie sich uber die einfach gestrichelte - Sehschärfe Linie erheben, bei hochgradiger $H$ wenigstens die - Ld - Linie. Am prägnantesten drückt sich aber die Schädigung der Augen durch die Kurzsichtigkeit dadurch aus, dass bei Myopie sowohl die Sehschärfe als sämmtliche Arten des Lichtsinnes unter der Normalen sich befinden, welche im Diagramm dureh die horizontale von 72 ausgehende doppelt gestrichelte Linie ausgedriickt wird. Hiermit ist doch festgestellt, dass die Ausbuchtung und Verduinnung der Sklera am hintern Pol, welche die Kurasichtigkeit zur Folge hat, kein so harmloser Vorgang ist, wie es von mancher Seite dargestellt wird. Auch wird hierdurch die Annahme einer mit der Ausbuchtung der Sklera verbundenen Zerrung und Dehnung der innern Augenhäute, wenigstens des Neuroepithels - des Sekretionsorgans des Sehpurpurs - recht wahrscheinIich gemacht. Noch wahrscheinlicher wird diese Annahme, wenn, wie wir gleich sehen werden, Sehschärfe und Lichtsinn schon beim Übergang von Emmetropie zu den niedersten Myopiegraden und gradatim mit ansteigendem Myopiegrade abnehmen. Hinsichtlich der Sehschärfe habe ich schon in meinen frühern Arbeiten nachgewiesen, dass eine Herabsetzung derselben schon vor Manifestwerden der Myopie und eines höhern Grades zu beobachten ist und nach Stationärbleiben der Myopie sich wieder bessert. Besonders hervorgehoben habe ich an letzterer Stelle, dass sich die Sehschärfe am in- und extensirsten bei den 15 jährigen, in welchem Lebensjahre auch die meisten kurzsichtig werden, verschlechtert, in den beiden obern Klassen, das ist in der Prima, sich wieder bessert. 
So in die Augen fallend nun in Tabelle II und Diagramm 1 die Übereinstimmung ist, welche zwischen den drei Prüfungsarten des Lichtsinnes besteht, so wird man sie bei den folgenden Darstellungen teilweise vermissen. Unterschieds- and Reizempfindlichkeit wurden nicht nur bei den einzelnen Individuen, sondern auch bei dem gleichen Refraktionszustande und gleichem Myopiegrad ziemlich verschieden gefunden.

Es ist dies a priori schon anzunehmen, da wir ja verschiedene Arten des Lichtsinnes: Reiz- und Unterschiedsempfindlichkeit und erstere teils peripher, teils zentral prüfen, es bat aber auch einen besondern Grund, auf den ich bei der folgenden Betrachtung zu sprechen kommen werde. Zunächst möchte ich hervorheben, dass die strengere Prüfung nach der Treitelschen Methode schon im allgemeinen eine geringere Wertigkeit des Lichtsinnes gibt als die Förstersche Methode.

Als typisches Beispiel dafür, dass die zentrale Lichtsinnprüfung nach Treitel eine strengere und auch die ausschlaggebendste ist führe ich folgendes an: Ein 29 Jahre alter Geistlicher, welcher auf dem rechten Auge nach Korrektur des einfachen myopischen As von $1,75 \mathrm{D} \mathrm{S}{ }^{6} / 8$ und auf dem linken Auge bei M. von $5 \mathrm{D}$ nur S $6 / 18$ hat, zeigte Lia beiderseits normal, Lic auch rechts normal, auf dem myopischen linken Auge aber nur $1 / 11$, dies bedurfte $10 \mathrm{~mm}$ Diaphragmadurchmesser zum Erkennen des weissen Quadrates statt $3 \mathrm{~mm}$.

In einzelnen Fällen ist die auffällig geringe Wertigkeit von Lic noch dadurch begründet, dass eine ausserordentlich langsame Dunkeladaption besteht. Wenn nun diese in der statistischen $\mathrm{Zu}$ sammenstellnng in besonders niedern Werten von Lic zum Ausdruck kommt, so geschieht dies gewiss nicht mit Unrecht, da die verlangsamte Adaption in ihrer Art auch eine Störung des Lichtsinnes, speziell der Reizempfindlichkeit ist. Langsame Dunkeladaption wurde besonders bei hochgradiger Ametropie und zwar nicht nur bei Myopie, sondern auch bei Hypermetropie gefunden, bei Myopie aber auch in mittlern und niedern Graden.

Auf Tabelle III, welche wir nun betrachten wollen, ist das Verhalten der Sehschärfe und des Lichtsinnes mit Ansteigen des Myopiegrades dargestellt. Bequemer noch verfolgen wir die folgenden Deduktionen am Diagramm 2, in welchem die Kurven gleiche Bedeutung haben, wie im Diagramm 1. Dem Verlauf der Kurven entnehmen wir nun, dass im allgemeinen wohl eine Abnahme der 
Tabelle II. Sehscharre und Lichtsinn abuangig rom Myopiegrade.

\begin{tabular}{|c|c|c|c|c|c|c|c|c|}
\hline \multirow{3}{*}{$\begin{array}{l}\text { Refraktion } \\
\text { bezw. Myopiegrad }\end{array}$} & \multirow{3}{*}{$\begin{array}{l}\text { Prozant- } \\
\text { satz }\end{array}$} & \multirow{3}{*}{ Sehsehingfe } & \multicolumn{6}{|c|}{ Durchschnittlicher Lichtsinn } \\
\hline & & & \multirow{2}{*}{\multicolumn{2}{|c|}{$\begin{array}{l}\text { Untersehieds- } \\
\text { empendlizhkeit } \\
\text { Ld }\end{array}$}} & \multicolumn{4}{|c|}{ Reizemplindichkeit $L i$} \\
\hline & & & & & \multicolumn{2}{|c|}{$\begin{array}{l}\text { nach } \\
\text { Forster a }\end{array}$} & \multicolumn{2}{|c|}{$\begin{array}{c}\text { nacil } \\
\text { Troitol }\end{array}$} \\
\hline Emmetropie & 59,5 & $90 / \mathrm{rz}_{2}$ & 15,5 & 85 & 1,9 & $80 / n a$ & 3,46 & $51 / 20$ \\
\hline Myopie 0,5 u. 0,75D & 3,8 & $74 / 72$ & $\mathbf{2 0}, 0$ & $62 / 79$ & 2,73 & $+77^{2}$ & $\begin{array}{l}3,400 \\
5,3\end{array}$ & $25 / 72$ \\
\hline$\Rightarrow \quad 1-1,75$ & 7,8 & $7 \pi / 79$ & 20,6 & $61 / 79$ & 2.56 & $52 / 72$ & 5,0 & $27 / 72$ \\
\hline $2-2,75$ & 9 & $67 / 78$ & 19 & $89 / 78$ & 2,52 & $54 / 42$ & 6,2 & $18 / 78$ \\
\hline 3 u. 3,5 & 3,1 & $73 / 72$ & 21.6 & $54 / 73$ & 2,8 & $45 / 79$ & 7,5 & $14 / 72$ \\
\hline $4 \mathrm{u}, 4,5$ & 3,1 & $12 / 27$ & 16,5 & $783 / 78$ & 3,0 & $36 / 72$ & 7,7 & $11 / 73$ \\
\hline$, \quad 5 \mathrm{u}, 5,5$ & + & $56 / 79$ & 17,3 & $74 / 78$ & 4,0 & $38 / 72$ & 10,28 & $7 / 72$ \\
\hline $6-9$ & 1,7 & $8 \pi / 72$ & 19 & & 4,0 & $8 / \% 2$ & & $5 / 72$ \\
\hline
\end{tabular}

Sehschärfe, die bei Emmetropie besser ist als normal, und ebenso eine A bnahme des Lichtsinnes mit der Steigerung des Myopiegrades stattfindet, doch ist die Abnahme von $\mathbf{S}$ und auch ron $L$ d (Unterschiedsempfindlichkeit) nicht stetig. Bei Myopie von drei und vier Dioptrien ergibt sich sogar wieder bessere Durchschnitssehschärfe und in besonders auffälliger Weise bei $M .4 \mathrm{D}$ bessere $L d, \mathrm{~S}$ und $I d$ werden sogar normal und besser als normal. Erst von fünf Dioptrien an sinkt die Sehschärfe erheblicher, $L d$ aber erst bei M. $6 \mathrm{D}$ und da nur mässig unter die normale, nämlich auf 69/72. Die Erklärung dieses scheinbaren Widerspruches ist aber eine sehr einfache. Die Myopen von 3 und 4 D sind meist Schüler höherer Klassen, bei denen die Myopie stationär geworden ist. Nachdem der myopische Prozess, das ist die mit Verlängerung des Bulbus in der sagittalen Achse verbundene Dehnung und Zerrung der innern Augenhäute aufhört, erholt sich das Auge, $\mathrm{S}$ und $L d$ werden wieder besser, während gegenteilig bei weiterem Fortschreiten des Myopiegrades durch Achsenverlängerung beide weiter abnehmen ' $/$, aber doch werden auch die Myopen von 3 bis $4 \mathrm{D}$ mit stationär gebliebener Myopie geschädigt, denn wie Tabelle III und Diagramm 2 ergeben, ihre Reizempfindlichkeit bessert sich durchschnittlich nicht, denn wir sehen sowohl bei Lia wie bei Lic nach unwesentlicher Besserung bei M. 1 und M.2D eine grosse stetige Abnahme mit Zunahme des Myopiegrades, so dass Lia bei M. 6-8 D nur mehr $1 / 4={ }^{18 / 72}$

1) Nachstehendes Beispiel zeigt, wie $L d$ bei M. 4,5 noch gut, bei M. 5,5 D schon erheblich schlechter sich findet und zwar bei gleicher Sehschärfo $=5 \%$ :

Links M. 5,5 D, Rechts M. $4 \mathrm{D}$,

$$
\operatorname{Ld}{ }^{45 / 72}, \quad 10 \% / 72 \text {. }
$$


$(4 \mathrm{~mm} \mathrm{DM})$, Lic mit $11 \mathrm{~mm}$ DM sogar nur mehr $<1 / 12=5 / 7_{2}$ des normalen beträgt. Auf diese ganz erschreckende Abnahme des Lichtsinnes - als zentralen $L$ geprüft - bei noch mässig hochgradiger Myopie möchte ich hier besonders hinweisen, da hiermit bekundet ist, wie intensiv der myopische Prozess den Lichtsinn schädigt. Dazu kommt noch, dass $L i c=5 / 72$ ein Durchschnitts-, kein Minimalwert ist, ich fand ihn nicht etwa nur vereinzelt, sondern relativ häufig und auch bei noch niedrigeren Myopiegraden, ja selbst $L i c=1 / 16$ und ${ }^{1 / 25}$ wurde von mir noch gefunden.

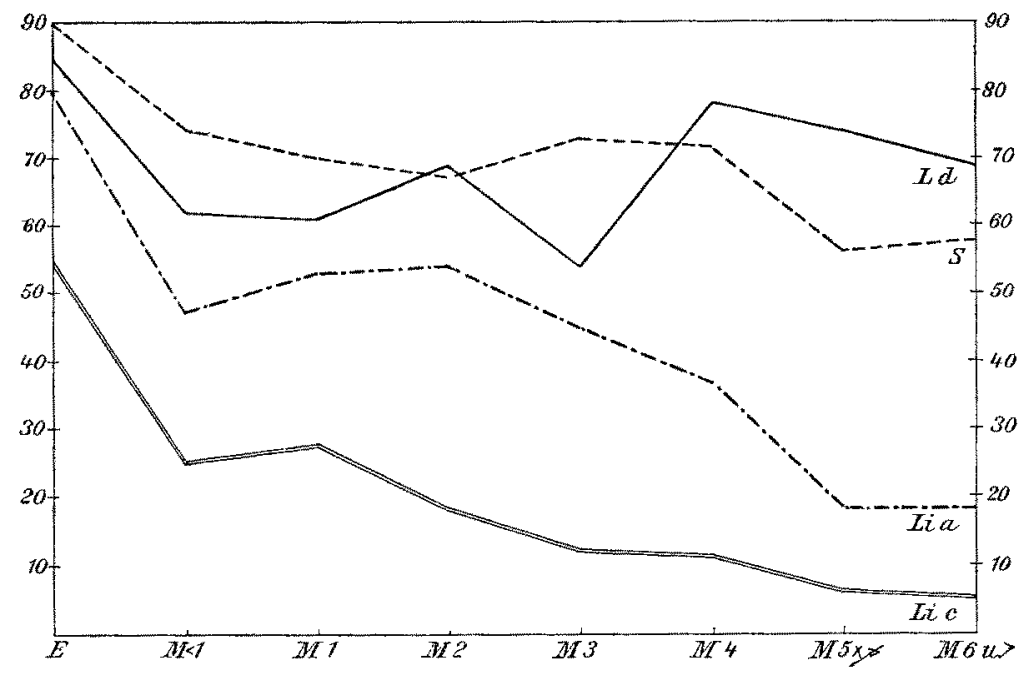

Fig. 2,

Einem weiteren naheliegenden Einwande muss ich hierbei aber begegnen, nämlich dem, dass, wenn bei M. 4 und $5 \mathrm{D}$ die Unterschiedsemptindlichkeit $L d$ durchschnittlich noch besser als normal, in einzelnen Fällen also noch erheblich besser ist, der myopische Prozess doch nicht so schädlich einwirken kann, als es bisher von mir hingestellt wurde. Eine teilweise Erklärung hierfür habe ich zwar eben schon gegeben, doch möchte ich noch anfügen, dass von mir für den

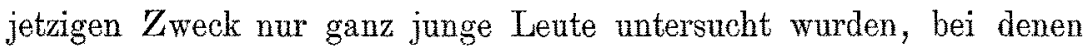
ebenso wie die Sehschärfe der Emmetropen in nicht seltenen Fällen bis zum doppelten Wert der normalen $12 / 6$ statt $\%$ steigt und die Grenze weit über ${ }^{6} / 6$ liegt, auch der Wert von $L d$, den ich mit $18=72 / 72$ bei schon ältern Leuten - zwischen 20 und 30 Jahren als normal festgestellt habe, bei jüngern Leuten eigentlich höher anzusetzen wäre. Ausserdem kommt noch in Berücksichtigung, dass 
bei Prüfung von $L d$ immer der Formensinn; i. e. die Sehschärfe eine Rolle mitspielt. Bei Prüfung von $L i$ prïfen wir dagegen die Reizschwelle, gewissermassen den reinen Lichtsinn, bei Lic am reinsten als zentralen ohne Zuhilfenahme jeglichen Formensinnes. Als Beweis hierfür gestatte ich mir auf das Seite 116 angeführte Beispiel hinzuweisen, indem bei M. $5 \mathrm{D}$ normaler $L d$ und sogar $L i a$ sich fanden, Lic aber erheblich herabgesetzt war.

Die Abnahme der Reizempfindlichkeit mit Zunahme des Myopiegrades ergibt sich somit aus Tabelle III und Diagramm 2 zur Evidenz. Die Zeichnung 2 lässt im besonderen noch ersehen, wie sowohl Sehschärfe als Lichtsinn schon bei den niedrigsten Myopiegraden erbeblich gegenüber der Enmetropie abnehmen. Ich habe auf diesen Umstand schon auf Seite 114 hingewiesen und angeführt, dass ich den Nachweis hinsichtlich der Sehschärfe schon in meinen frühern Arbeiten erbracht habe. Nunmebr scheint es mir auch für den Lichtsinn nachgewiesen und zwar für alle drei Versuchsarten, also auch für die Unterschiedsempfindlichkeit, für welche Samelsohn ${ }^{1}$ ), der mit der Massonschen Gradscheibe untersuchte, eine Verminderung nur bei höhern Graden der Ametropie gefunden hat - die Grenze läge etwa bei 3 Dioptrien. - Ich glaube nun nicht zu irren, wenn ich annehme, dass der starke Abfall von $\mathbf{S}$ und L. von Emmetropie zu den geringsten Myopiegraden $(<1 \mathrm{D})$ dadurch begründet ist, dass hier die Evolution des myopischen Prozesses am störendsten einwirkt und noch keine Erholung mit dem Stationärwerden der Myopie wie bei den Graden von 1 bis $4 \mathrm{D}$ eingetreten ist.

An ein paar Beispielen vermag ich zu zeigen, wie mit Evolution der Myopie Verschlechterung der Unterschiedsempfindlichkeit eintritt, teils mit Abnahme, teils bei Gleichbleiben der Sehschärfe, ja sogar trotz Besserung derselben:

1. F. weiblicher Zögling

$$
\begin{array}{cccccc}
\text { mit } 13 \text { Jahren } & \text { H. } 0,25 \mathrm{~S} 8 / 6 & \left.L d<{ }^{8 / 12}=82 / 72^{2}\right) \\
n & 15 & \text { M. } 0,25 \mathrm{~S} 6 / 6 & \left.n<6 / 18={ }^{6} / 72^{3}\right)
\end{array}
$$

2. Gr. weiblicher Zögling

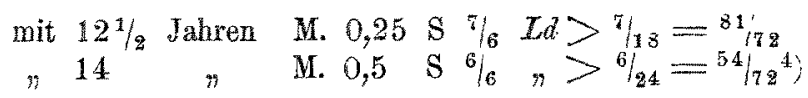

1) Loc. cit.

2) 8) 4) Vgl. S. 109. 
3. E. weiblicher Zögling

$$
\begin{array}{cclccc}
\text { mif } 11 \text { Jahren } & \text { H. } 0,25 \mathrm{~S} 6 / 6 & \text { Ld } 6 / 12=108 / 72 \\
n & 12 & \text { M. } 1 & \mathrm{~S} 6 / 6 \quad " 6 / 18=72 / 78
\end{array}
$$

4. Th. weiblicher Zögling

$$
\begin{array}{ccccccc}
\text { mit } 10 & \text { Jahren } & \text { H. } 0,5 & \text { S } & 7 / 6 & L d<7 / 12=92 / 72 \\
" 15 /_{2} & \# & \text { M } & 0,25 & \text { S } & 7 / 6 & \#<7 / 8=65 / 72
\end{array}
$$

5. H. weiblicher Zögling

$$
\begin{array}{cccccccc}
\text { mit } & 12 & \text { Jahren } & \text { H. } 0,25 & \mathrm{~S} & 6 / 6 & \mathrm{Ld} & 6 / 12=108 / 72 \\
" 14 & 14 & \text { M. } 0,5 & \mathrm{~S} & 7 / 6 & 7 & 7 / 24=54 / 72
\end{array}
$$

Ein sechstes Beispiel zeigt, wie sich, nachdem die Myopie stastionär geworden, bei Gleichbleiben von S die Unterschiedsempfindlichkeit wieder bessert und sogar sehr erheblich bessert:

6. H. weiblicher Zögling

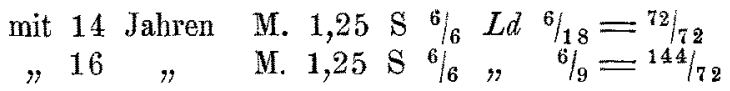

Endlich mögen noch vier Fälle mit etwas höhern Myopiegraden angeführt werden, bei denen die Reizempfindlichkeit $L i$ besonders gelitten hat, während die Unterschiedsempfindlichkeit $L d$ gut und sogar sehr gut geblieben ist, bezw. sich wieder gebessert hat.

7. M. weiblicher Zögling

mit 16 J. M. 6 S $5 / 6$ Ld $5 / 18=72 / 72$ Lia $21 / 2=54 / 72$ Lio $11=5 / 72$

8. Sch. weiblicher Zögling

mit 16 J. M. $4 \mathrm{~S}^{2 / 6}$ Ld $<{ }^{2 / 12}={ }^{92} /{ }_{72}$ Lia $5^{1 / 2}=10 / 72$ Lic $12=4 / 72$

9. R. männlicher Zögling:

mit 18 J. M. $4,5 \mathrm{~S} 8 / 6$ Ld $8 / 18=108 / 72$ Lia $3={ }^{36 / 72}$ Lic $6={ }^{18 / 72}$

10. E. männlicher Zögling

mit 17 J. M. $6 \mathrm{~S} \mathrm{t}^{4 / 6}$ Ld ${ }^{4} / 12=108 / 72$ Lia $6=8 / 72$ Lic $15=3 / 72$

Auf weitere Details einzugehen muss ich mir, um nicht zu ermüden, versagen. Ich kann hier nur versichern, dass die scheinbaren Ausnahmen bei genauer Analyse die Regel nur bestätigen.

Ferner könnte mir noch entgegengehalten werden, dass nach der ersten Querspalte von Tabelle III selbst emmetropische Augen keinen voll normalen zentralen Lichtsinn zeigen, nicht $3 \mathrm{~mm} \mathrm{D.} \mathbf{M}$. $={ }^{72} / 72$, sondern nur $3,46 \mathrm{~mm}$ D. M. $={ }^{54} / 72$. Darauf erwidere ich, dass wie die folgende Tabelle IV ergibt, in der untersten Altersstufe, die nur Mädchen einschliesst, Iic besser als normal $={ }^{96} / 72$ gefunden wurde und dass überhaupt bei den Mädchen der durchschnittliche Lichtsinn für Emmetropen nach Querspalte 1 von Tabelle II genau $3 \mathrm{~mm}$ D. M. $={ }^{72 / \%}$ entspricht. Bei den Knaben, die unter 
weniger guten hygienischen Verhältnissen stehen und mehr hereditär belastet sind, so dass wir bei ihnen auf viel mehr Myopen stossen, ist schon, wenn sie noch Emmetropen sind, der Lichtsinn etwas geschädigt. Ich bin auch fest ïberzeugt, dass, wenn wir emmetropische Knaben und Mädchen nach vierwöchentichem rationellex Landaufenthalte untersuchen, erstere sicher durchschnittlich Lic normal und letztere besser als normal haben. Ich kann hierfiur auch ein Beispiel anfithren. Die Schülerin E. der VIII. Klasse zeigte

$$
\begin{aligned}
& \begin{array}{lll}
\text { mit } 141 / 2 J, & \text { H. } 0,25 \mathrm{D} S=9 / 6 & \text { Ld } 9 / 18=72 / 72 \\
\text { Lia } 1,25=190 / 72 & \text { Lic } 2,75=861
\end{array} \\
& \text { nit } 161 / 2 \text { J. H. } 0,25 \mathrm{D} \mathrm{S}=\pi / 6 \quad L d 7 / 86=36 / 72 \\
& \text { Lit } 2=72 / 72 \quad \text { Lit } 3,25=62 / 72
\end{aligned}
$$

nachdem sie unmittelhar vorher angestrengt an einer feineren Stickerei gearbeitet hatte. Nachdem die Schülerin längere Zeit ausgeruht hatte, war $\mathrm{S} 8 / 6$ und $L$ durchweg normal. Ein weiteres Beispiel zeigt, wie Spasmus accommodationis ebenso wie Überanstrengung. der Accommodation die Reizempfindlichkeit herabsetzt:

Weiblicher Zögling

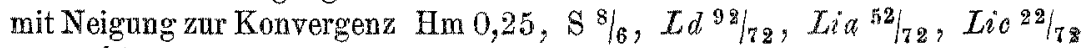
sehielen

ophthalmoskopisch H. $1,5 \mathrm{D}$.

Ausser nach Geschlecht, Refraktion und Myopiegrad habe ich das Verhalten von S, $L a$ und $L i(a$ und $c)$ auch nach fünf Altersstufen in Betracht gezogen und zwar umfasst:

I die Stufe von 9 und 10, II von 10 bis 12 (Sexta und Quinta); III von 12 bis 14 (Quarta und Untertertia), IV von 14 bis 17 (Obertertia und Secunda), $V$ von 18 und 19 Jahren (Prima).

In dieser Tabelle ist auch der Refraktionszustand im Prozentverhältnisse angegeben, wobei ich insbesondere das der Myopen durch fetten Druck hervorgehoben - zu bericksichtigen bitte. Wir sehen nun, wenn wir gleichzeitig Diagramm 3 ins Auge fassen, dass von der untersten bis zur obersten Altersstufe Sehschärfe und Unterschiedsempfindlichkeit wenig abnehmen, $S$ sinkt nicht einmal unter die normale ${ }^{1}$, $L d$ nur in der V. obersten Altersstufe (Prima), wo der Prozentsatz der Myopen schon ein sehr hoher ist, nur wenig unter die normale: auf ${ }^{65 / z q}$. Die Reizempfindlichleit und zwar so-

1) So gute Sehschärien in den zwei obersten Gymnasialklassen wird man selten finden, nur dort, wo ebenso gute hygienische Verhältnisse bestehen, wie in den beiden von mir untersuchten Instituten (Internaten), die Reizempfindichkeit hat freilich anch hier gelitten. 
Tabelle IV. Sehschärfe und Lichtsinn nach Altersstufen.

\begin{tabular}{|c|c|c|c|c|c|c|c|c|c|}
\hline \multirow{3}{*}{ Altersstufe } & \multirow{3}{*}{ Zahl } & \multirow{3}{*}{ 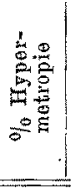 } & \multirow{3}{*}{ 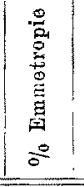 } & \multirow{3}{*}{ 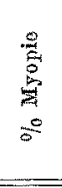 } & \multirow{3}{*}{ 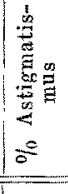 } & \multirow{3}{*}{$\begin{array}{c}\text { Durck- } \\
\text { schnitt- } \\
\text { Iiche } \\
\text { Seh- } \\
\text { sehahre }\end{array}$} & \multicolumn{3}{|c|}{ Durchschnittlicher Lichtsinn } \\
\hline & & & & & & & \multirow{2}{*}{$\begin{array}{c}\text { Onter- } \\
\text { sohieds- } \\
\text { empfindlich- } \\
\text { kait } L i\end{array}$} & \multicolumn{2}{|c|}{ Reizempfindlienkeit $L i$} \\
\hline & & & & & & & & $\begin{array}{c}\text { nash } \\
\text { Forrster Lia }\end{array}$ & $\begin{array}{c}\text { nach } \\
\text { Treitel } L i c\end{array}$ \\
\hline I. $\left.\begin{array}{r}9 \text { und } \\
10 \text { Jahre }\end{array}\right\}$ & 20 & 45 & 45 & 10 & - & $81 / 72$ & $14,890 / 72$ & $1,5 \% 5^{119 / 72}$ & $2,86 \quad 96 / 72$ \\
\hline II. $\left.\begin{array}{lll}10 & \text { bis } \\
12 & \text { Jahre }\end{array}\right\}$ & 67 & 24 & 49 & 12 & 15 & $80 / 72$ & $13,596 / 72$ & $2,25 \quad 63 / 72$ & 3,5 \\
\hline III. $\left.\begin{array}{lll} & 12 & \text { bis } \\
& 14 & \text { Jahre }\end{array}\right\}$ & 73 & 22 & 45 & 16,5 & 16,5 & $83 / 72$ & $17,673 / 72$ & $2,16 \quad 67 / 72$ & $4,16 \quad 8 \% / 72$ \\
\hline IV. $\left.\begin{array}{ll}14 & \text { bis } \\
& 17 \text { Jahre }\end{array}\right\}$ & 81 & 8,6 & 51,4 & 33,0 & 7,0 & $78 / 72$ & $72 / 72$ & 2,2 & 4,7 \\
\hline $\left.\begin{array}{l}18 \text { and } \\
19 \text { Jahre }\end{array}\right\}$ & 40 & - & 17,5 & 72,5 & 10,0 & $74 / 79$ & $85 / 72$ & 2,7 & 7,3 \\
\hline
\end{tabular}

wohl Lia und Lic, welche in der jüngsten Altersstufe sehr gut, weit über normal, waren, sinken schon in der zweiten Altersstufe und zwar ganz enorm und erreichen, nachdem sich Lia in der III. und IV. Altersstufe wieder etwas gehoben, Lic gleichmässig abgenommen hat, in der V. Altersstufe ein sehr niedriges Niveau, Lic ist sogar auf $12 / 72=1 / 6$ der normalen gekommen. Ein Blick auf die Prozentziffern der Myopen gibt uns auch hier wieder die Ursache der Verschlechterung des Lichtsinnes an. Diese Prozentziffer erhöht sich anfänglich in Altersstufe II und III von 10 auf 12 bezw. 14 Jahre nur wenig, steigt aber dann in IV und $V$ auf 33 und $72,5 \%$ rapid $^{1}$. Dementsprechend sehen wir im Diagramm 3 die gestrichelt-punktierte und doppelt ausgezogene Kurve - Li ${ }^{-}$und Lic - mit der einfach gestrichelten, welche hier nicht die Sehschärfe, sondern den Prozentsatz der Myopen bedeutet, ziemlich gleichmässig abfallen. Die Kurve von $L d$ - einfach ausgezogen - senkt sich dagegen analog der Sehschärfe nur sehr allmählich. Die Sehschärfekurve ist im Diagramm 3 weggelassen, um das Bild nicht zu verwirren.

Da Zunahme der Kursichtigen mit Erhöhung der Altersstufe

1) Das plötzliche stärkere Ansteigen des Myopenprozentsatzes in Altersstufe IV kommt, wie ich in meiner letzten Arbeit in v. Graefe's Arch. f. Ophthalm. S. 560 1. c. S. 110 gezeigt habe, davon her, dass hauptsächlich mit 15 Jahren die erworbene Myopie auftritt. Dagegen kommt der noch höhere Prozentsatz der Myopen in der V. Altersstufe nicht davon her, dass noch mehr Zöglinge kurzsichtig werden, da Eintritt der Myopie nach dem 18. Lebensjahre von mir nur höchst selten beobachtet wurde, sondern daher, dass viele Nichtmyopen nach Absolvierung der Secunda austreten, 
zusammenfällt, so nimmt auch die punktiert-gestrichelte und doppelt ausgezogene Kurve im Diagramm 2 und 3 ähnlichen Verlauf und bitte ich beim Vergleich derselben insbesondere den anfänglichen gemeinsamen steilen Abfall der beiden Li-Kurven zu beachten, wodurch die Schädigung des Lichtsinnes als Reizempfindlichkeit schon bei Erolution der Myopie und in den niedersten Myopiegraden $(M<1 D)$ zum Ausdruck kommt. Wenn zwischen dem Kurvenverlauf auf Didgramm 2 und 3 keine grössere Übereinstimmung herrscht, so liegt die Ursache darin, dass in dem Diagramm 3 für die Altersstufen auch die Nichtkurzsichtigen mit aufgenommen sind, dass also beson. ders gute nicht myopische Augen in einer Altersstufe bessere Durchschnittswerte für $L i$ geben. Dies trifft auch für $\mathrm{S}$ und $L d \mathrm{zu}$, daher bildet auch die gute durchschnittliche Sehschärfe und Unterschiedsempfindlichkeit der vier unteren Altersstufen, wie sie Tabelle IV zeigt, keinen Widerspruch zwischen Tabelle III und IV.

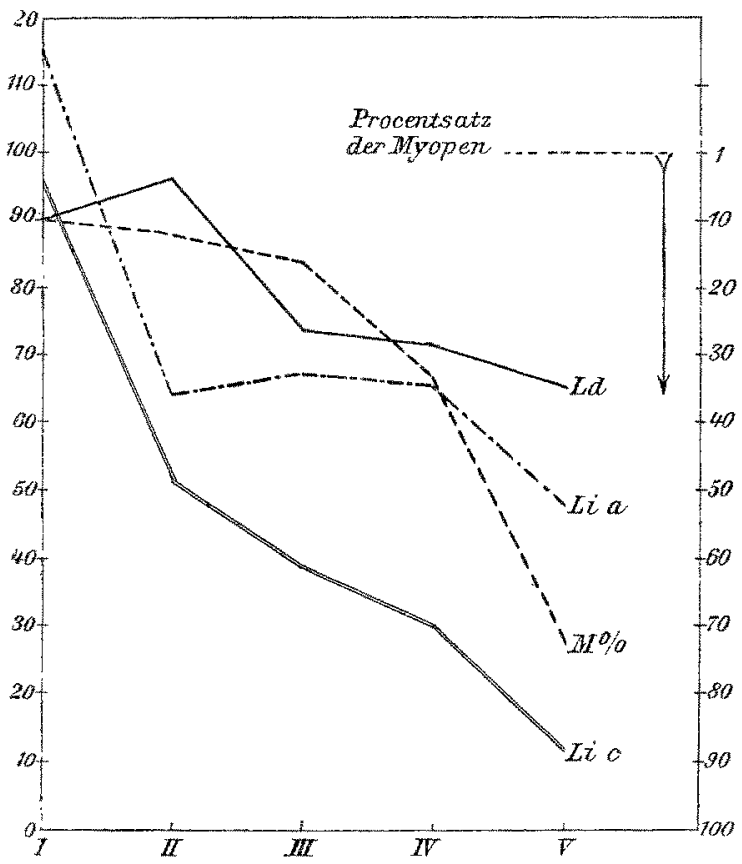

Fig. 3,

Noch besser und wohl alle Zweifel schlagend tritt die Abnahme des Lichtsinnes mit aufsteigender Altersstufe zutage, wenn man den Prozentsatz der Zöglinge mit normalem Lichtsinn, nach den drei Arten als Lu, Lia und Lic geprüft, mit dem Prozentsatz der 
Tabelle V. Abnahme des Prozentsatzes normalen Lichtsinnes nach Altersstufen.

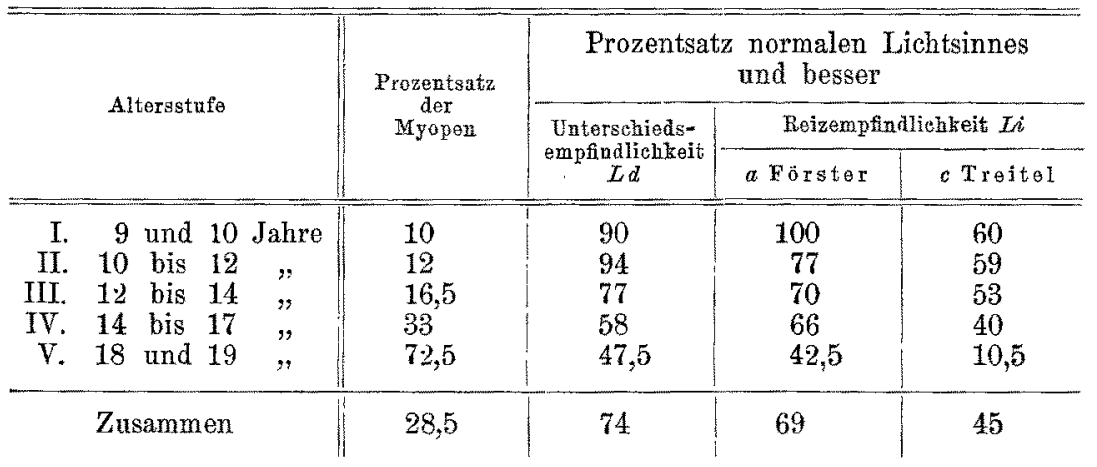

Myopen in Gegensatz bringt, wie es in Tabelle $V$ geschehen ist, die keiner weitern Erklärung bedarf. Noch in die Augen fallender zeigt Diagramm 4, dass der Lichtsinn mit den zunehmenden Schuljahren

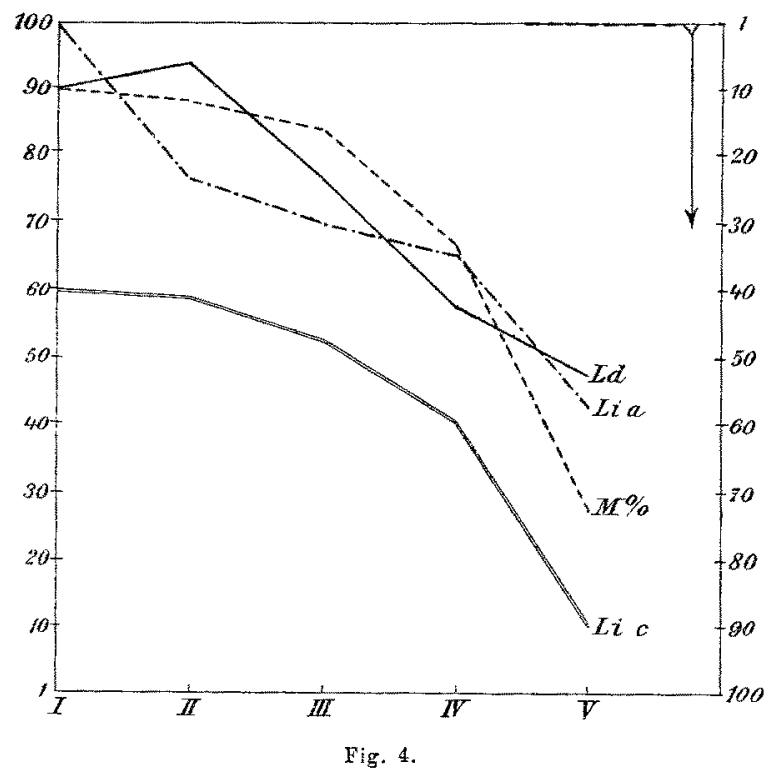

und proportional der Zunahme der Kurzsichtigen beträchtich abnimmt. Das Abfallen der drei Lichtsinnkurven und das Aufsteigen des Myopiegrades - im Diagramm 4 ebenso wie im Diagramm 3 in umgekehrter durch die Pfeilrichtung angegebener Richtung aufsteigend - ist hier doch ein überraschend gleichmässiges. Nur das auf den vier andern Tabellen und drei ersten Diagrammen hervorgetretene Ver- 
halten der drei Untersuchungsarten zu einander in dem Sinne, dass $L d$ am wenigsten, Lic am meisten geschädigt erscheint, erleidet hier eine Ausnabme, indem in der vierten Altersstufe $L d$ stärker geschädigt erscheint als Lia - nur $58 \%$ haben normalen $L d$, dagegen $66 \%$ normalen Lit -, die punitiert-gestrichelte Kurve liegt hier über der einfach ausgezogenen. In der $V$. Altersstufe, welche nur männliche 18- bis 19jährige Zöglinge enthält, stellt sich das gewöhnliche Verhältnis wieder her, indem der Prozentsatz der Zöglinge mit normalem $L d 47,5$, deren mit normaler Lia nur $42,5 \%$ beträgt. Der Prozentsatz der Zöglinge mit normaler Lic ist hier sogar auf $10,5 \%$ gesunken.

Ziehe ich endlich an Stelle der Aitersstufen die verschiedenen Refraktionszustände in Betracht, so ergibt sich:

$L d$ ist normal und besser

bei Emmetropie und geringer Hypermetropie in $83 \%$

bei Myopie uur in $55 \%$

(bei Ash in 77, bei hochgradiger $H$. in 10, Asm in $22 \%$ ).

Lia ist normal und besser

bei Emmetropie und gexinger Hypermetropie in $92 \%$

bei Myopie nur in $40 \%$

(bei $A s h$ in 81 , bei hochgradiger $\mathrm{H}$. in 10 , bei $A s m$ in $33 \%$ ).

Lic ist normal und besser

bei Emmetropie und geringer Hypermetropie in $66 \%$

bei Myopie nur in $20 \%$

(bei Ash in 36, bei hochgradiger $\mathrm{H}$. in 0 , bei Asm in $10 \%$ ).

Wir sehen also, dass der Lichtsinn bei Myopie nicht nur dem Grade, sondern auch dem Prozentsatze nach überraschend häufig geschädigt wird, am häufigsten und intensivsten als zentrale Reizempindlichkeit, in dem nur $20 \%$ der Myopen normalen Lic haben. Diagramm o, aus vorstehenden Daten zusammengestellt, veranschaulicht die von der Refraktion abhängige Abnahme des Prozentsatzes nor malen Lichtsinnes in einer gewissen Übereinstimmung mit Diagramm 4, da mit ansteigender Altersstufe ebenfalls der Prozentsatz normalen Lichtsinnes abnimmt. Die intensive Schädigung des Lichtsinnes bei Myopie ging aus Tabelle II und Diagramm 1 hervor und tritt noch drastischer zutage, wenn ich nochmals hervorhebe, dass in einzelnen Fällen Lic bis auf $1 / 16$ und selbst $2 / 25$ des normalen herabsinkt.

In der unerwartet hochgradigen Schädigung des Lichtsinnes bei den Myopen darf ich doch sicher eine Bestätigung meiner schon friher und auch hier wiederholt geäusserten Anschauung erblicken, 
dass die dem Eintritt und der Progression der Myopie zu Grunde liegende Verdünnung und Ausbuchtung der Sklera mit Dehnung und Spannung der beiden innern Augenhäute, insbesondere des Neuroepithels und seines Pigmentes einhergeht.

Die besonders in Erscheinung tretende Schädigung des zentralen Lichtsinnes könnte vielleicht als irrige Beobachtung angefochten werden, indem der Versuch nicht richtig angeordnet oder die Priffung im Verhältnis zu den beiden andern Methoden der Lichtsinnprïfung zu schwer, die Reizschwelle zu hoch angelegt sei. Ich kanu dagegen anführen, dass $33 \%$ der weiblichen Zöglinge, also ein volles Drittel übernormalen $\mathrm{Lic}$ hatten. Die Probe ist also weder absolut noch relativ zu schwer, sondern sie bildet eine einen sehr feinen Ausschlag gebende Methode.

Die unterste Querreihe von Tabelle $V$ lässt die Feinheit der drei Prïfungsmethoden im umgekehrten Verhältnis zum Prozentsatze der Zöglinge mit normalem Lichtsinn und besserem in obigem Sinne gut ersehen, indem sich bei $74 \%$ normaler und übernormaler $L d$, bei $69 \%$ ebensolcher Lia und nur bei $45 \%$ normaler und übernormaler Lic ergibt. Ungefähr dieselbe Abstufung für die drei Arten der Lichtsinnprüfung zeigt die Zusammenstelluug der männlichen und weiblichen Zöglinge in Tabelle I, nämlich 74 für $L d, 65$ für $L i a$ und 45 für Lic in $Z$ weiundsiebzigstel als Durchschnittswert. Eine weitere Betrachtung möge hier noch Platz finden. Stellte ich bei den männlichen Zöglingen den in den verschiedenen Klassen gefundenen Lichtsinn aller drei Gattungen zusammen, so fand ich die in Tabelle VI aufgeführten Prozentverhältnisse von Zöglingen mit normalem und übernormalem Lichtsinn.

Tabelle VI.

\begin{tabular}{|c|c|c|c|}
\hline \multicolumn{2}{|r|}{ Klasse } & $\begin{array}{l}\text { Prozentsatz mit normalem } \\
\text { and úbernurmalem Lichtsiin }\end{array}$ & $\begin{array}{l}\text { Prozentsatz } \\
\text { der Myopischgewurdenen }\end{array}$ \\
\hline I. & Sexta & 67,5 & 2.23 \\
\hline II. & Quinta & 54,4 & 5,78 \\
\hline IIII. & Quarta & 45,0 & 9,52 \\
\hline IV. & Untertertia & 57,6 & 8,82 \\
\hline V. & Obertertia & 32,7 & 15,72 \\
\hline VI. & Untersecunda & 36.3 & 5,32 \\
\hline VII. & Obersecunda & 36,7 & 4,9 \\
\hline VIII. & Unterprima & 27,6 & 2,44 \\
\hline IX. & Oberprima & 46,2 & 0 \\
\hline
\end{tabular}

Vergleichen wir nun die 1. Reihe der Tabelle VI mit der 2. Reihe, die den Prozentsatz der Myopischgewordenen nach Klassen 
gibt $^{3}$ ), so ist doch eine Übereinstimmung beider Reihen in dem Sinne unverkennbar, dass die Klassen, in welchen die wenigsten kurzsichtig werden, auch der höchste Prozentsatz von Zöglingen mit normalem und übernormalem Lichtsinn haben, und dass anderseits die V. Klasse (Obertertia), in der nach meinen Untersuchungen die meisten kurzsichtig werden, auch der niederste Prozentsatz yon Zöglingen mit normalem und ibernormalem Lichtsinn sich findet. Besonders tritt dieser Zusammenhang bei der Cnterschiedsempfindlichkeit hervor, da hier in der V. Klasse nur $8,3 \%$ der Zöglinge normalen $L d$ und besser haben. (Der noch geringere Prozentsatz der VIII. Klasse steht mit meinen Schlussfolgerungen nicht in Widerspruch, denn in dieser Klasse befanden sich bei den Lichtsinnuntersuchungen $80 \%$ Myopen, $10 \%$ mit Asm und nur 10\% Emmetropen, und erklärt sich der geringe Prozentsatz normalen Lichtsinnes als Spiel des Zufalls in einfachster Weise.) Der geringe Prozentsatz normalen Lichtsinnes von der V. Klasse aufwärts ist überhaupt durch Progression der Myopie und die grosse Myopenzahl bedingt.

Die Prozentverhältnisse der Zöglinge mit normalem und übernormalem Lichtsinn ( $L d$, Lia und $L i c$ ) bei den iibrigen Refraktionszuständen entsprechen im grossen und ganzen dem, was Tabelle II und Diagramm 1 hinsichtlich des durchschnittlichen Lichtsinnes ergeben. Der geringe Prozentsatz mit normalem und übernormalem Lice bei Emmetropie (nur 66\%) deutet uns an, bezw. bestätigt das oben Ausgesprochene, dass die zentrale Reizempfindlichkeit am frühesten und leichtesten geschädigt wird.

Bei hypermetropischem Astigmatismus finden wir im allgemeinen nächst Emmetropie den besten Lichtsinn und die höchste Prozentziffer mit normalern Lichtsinn, bei myopischem Astigmatismus am wenigsten, noch weniger als bei reiner Myopie aus dem gleichen Grunde, den ich 8. 112 angefürt habe. Charakteristisch für die Prüfungsart des Lichtsinnes ist endlich, dass bei Asm sich mehr Zöglinge mit normalem Lia finden als mit normalem $L d$ (33 gegen $22 \%$, weil eben bei letzterer Prüfung der Formensinn, der bei $A s$ auch bei möglichst guter Korrektur herabgesetzt ist, mit beansprucht wird. Aus gleichem Grunde ist auch bei Ash der Prozentsatz normaler Lia grösser als der normalen $L d$ ( 81 gegen $77 \%$ ), doch ist hier der Unterschied viel geringer als bei Asm. Diese Erscheinung tritt besonders im Diagramm $\check{~ z u t a g e, ~ i n d e m ~ s i c h ~ h i e r ~ d i e ~ p u n k t i e r t-~}$

1) Loe. cit. v. Graefe's Arch. f. Ophthalm. Bd. XLVI. 3. 
gestrichelte Kurve bei Ash sowohl wie bei Asm über die einfach ausgestrichene erhebt. Dass endlich bei hochgradiger H. der Lichtsinn ebenso wie die Sehschärfe sehr schlecht ist, ist, wie ich ebenfalls schon hervorgehoben, nicht verwunderlich, da wir es hier mit in der Entwicklung zurückgebliebener Augen zu tun hatten.

Einem weitern Einwande muss ich schliesslich noch begegnen, nämlich dem, dass nach den allgemeinen Erfahrungen die Abnahme des Lichtsinnes doch nicht erbeblich ins Gewicht falle, wenigstens nicht als Mangel oder Gebrechen empfunden werde. Ich kann diesem

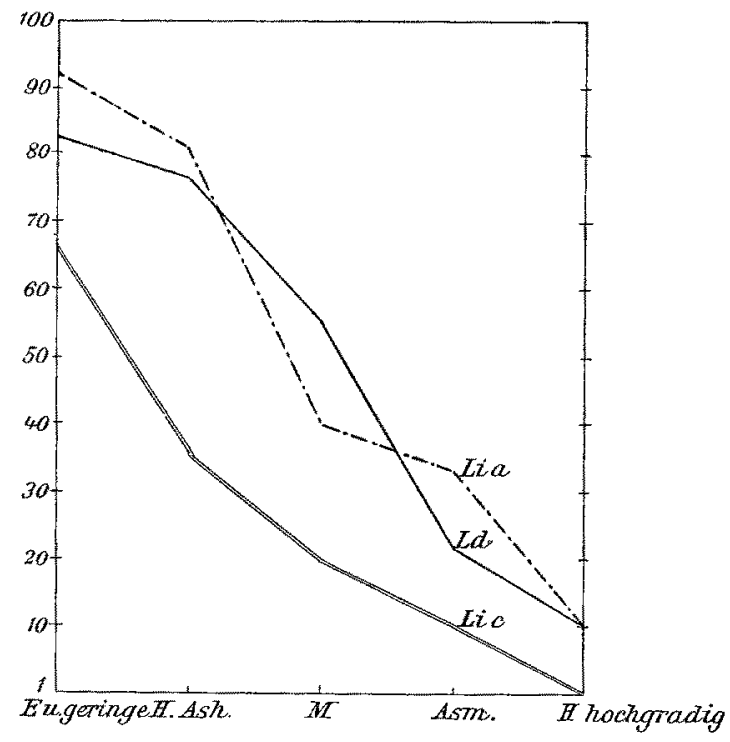

Fig. 5.

Einwande folgendes entgegenhalten: Schon $\mathrm{H} . \mathrm{Cohn}^{1}$ ) hat im Jahre 1884 bei abnehmender Beleuchtung enorme individuelle Differenzen der Sehschärfe gefunden, und die von Carp und Dörinkel auf Veranlassung Schmidt-Rimplers gemachten Versuche haben ergeben, dass mit fortschreitendem Alter die Sehschärfe bei abnehmender Beleuchtung bedeutende Einbusse erleidet. Eine bekannte Tatsache ist es ja, dass ältere Leute in der Dämmerung und Dunkelheit erheblich and in störender Weise schlechter sehen, um wie viel mehr wird die Verschlechterung der Sehschärfe bei abnehmender Beleuchtung sich geltend machen, wenn der Lichtsinn in der

1) Gohn, H., Untersuchungen über die Sehschärfe bei abnehmender Beleuchtung. Arch. f. Augenheilk. Bd. XIII. 
Jugend schon so erheblich geschädigt wurde, wie ich es im Voransgehenden nachweisen konnte. Denn es ist wohl von vornhinein klar, dass die unverhältnismässige Abnahme der Sehschärfe bei abnehmender Beleuchtung im Alter durch Schädigung des Lichtsinnes bedingt ist. Diese Nachtblindheit älterer Myopen äussert sich nicht bei Nahbeschäftigung - hochgradige ältere Myopen vermögen sogar bei sehr schlechter Beleuchtung noch feinen Druck zu lesen. Sie vermögen dies wegen der grossen Netzhautbilder, die sie infolge der starken Annäherung erhalten, auch ist bei ihnen die Unterschiedsempfindlichkeit weniger herabgesetzt als die Reizemptindlichkeit. Die Nachtblindheit der Myopen tritt vielmehr bei der Orientierung in der Dämmerung und in der Dunkelheit störend herwor. Die Hypermetropen und Presbyopen haben dagegen für die Beschäftigung in der Nähe grosses Lichtbedürnis, nicht aber wegen Abnahme des Lichtsinnes, sondern um eine enge Pupille zu belzommen. Aitere höhergradige Hypermetropen, welche sich ebenfalls im Dunkeln schlecht zurechtfinden, haben; wie Tabelle II und $V$ sowie Diagramm 1 und 5 zeigten, ebenfalls geringe Reizempfindlichkeit, aber als angeborenen Zustand, nicht als erworbenen, wie die Myopen. Bei den Hypermetropen ist überdies der schlechte Lichtsinn für die Orientierung noch störender, weil sie eine engere Pupille haben als die Myopen. - Wie sich übrigens Verschlechterung des Lichtsimnes schon bei jungen Leuten störend macht, dafür lieferten mir die von mir untersuchten männlichen Zöglinge den Beweis. War bei meinen Sehschärfeprüfungen das Wetter trübe, der Himmel mit Regenwolken bedeckt, so fand ich bei einer grossen Zahl derselben und zwar in erster Linie bei den Myopen die Sehschärfe so erheblich abgemindert, dass ich die Untersucbung abbrechen und auf besseres Wetter verschieben musste. Die Jünglinge mit Emmetropie und geringer Hypermetropie und $\mathbb{S}>1$ sehen dagegen auch bei schlechter Tagesbeleuchtung gut, und bei den besser sehenden und wenig Mropen zählenden Mädchen war ich von der Tagesbeleuchtung nicht so sehr abhängig, wie bei den männlichen Studierenden ${ }^{1}$.

1) Da Abnahme des Lichtsinnes bezw. der Reizempfindlichkeit sich als unverhältnismässige Abnahme des Behvermogens bei herabgesetzter Beleuchtung aussert, habe ich in dem Texte $z u$ meinen Lichtsinntafeln in beregtem Sinne vorgeschlagen, die Reizempfindlichkeit $L i$ dadurch zu bestimmen, dass die Sehschärfe des zu Untersuchenden mit der weissen Snellenschen Tafel Nr. 1 bei herabgesetzter Beleuchtung geprift und der erbaltene Wert mit dex in gleicher Weise geprüften Sehschärfo des Untersuchenden, dessen Augen natürlich normale 
Das Ergebnis meiner Untersuchungen fasse ich zum Schluss in folgendem zusammen:

Ich habe gezeigt,

1. dass die weiblichen $Z$ öglinge, bei denen sich weniger Kurzsichtige finden und der durchschnittliche Myopiegrad geringer ist, bessere durchschnittliche Sehschärfe und bessern Lichtsinn haben, als die männlichen (Tabelle I);

2. dass unter den verschiedenen Refraktionszuständen der Lichtsinn bei den Myopen am schlechtesten (Tabelle II und Diagramm 1) und der Prozentsatz der Kurzsichtigen mit normalem Lichtsinn gegenüber dem der Emmetropen ausserordentlich gering ist (Tabelle V und Diagramm 5). (Bei Myopie ist überdies gegenüber der Emmetropie und geringgradigen Hypermetropie nicht nur der Lichtsinn, sondern auch die Sehschärfe durchschnittlich unternormal (Tabelle II, Diagramm 1);

3. dass der Lichtsinn ebenso wie die Sehschärfe mit Zunahme des Myopiegrades (Tabelle III und Diagramm 2) und mit aufsteigender Altersstufe bezw. Schulklasse (Tabelle IV und V und Diagramm 3 und 4) schlechter gefunden wird, bei letztern beiden wesentlich nur deshalb, weil sich mit ihrem Ansteigen der Prozentsatz der Kurzsichtigen und der Grad der Myopie erhöht;

4. dass Schädigung des Lichtsinnes schon mit Evolution des myopischen Prozesses und brei den niedrigsten

Sehschärfe und normalen Lichtsinn haben müssen, verglichen wird. Da graduelle Verdunkeling des Zimmers schwer herzustellen ist - Wolffbergs Seidenapparat würde sich allerdings gut dazu eignen - und die auf einem Vergleiche beruhende Feststellung doch nicht sehr exakt ist, habe ich die Untersuchung von $L_{i}$ mit dem Försterschen Photometer vorgezogen. Auch auf die weitern Modifikationen der Lichtsinnprüfung, die sich mit meinen Lichtsinntafeln vornehmen lassen und die $\operatorname{Schwar}^{\mathbf{x}}$ ) besonders ausgebildet hat, habe ich mit Rücksicht auf mein Untersuchungsmaterial, welches ein recht einfaches Vorgehen erforderte, verzichtet.

Eingehendere Arbeiten über das Abhängigkeitsverhältnis der Sehschärfe von der Beleuchtungsintensität verdanken wir, wie ich hier nicht unerwähnt lassen möchte, Uhthoff') und besonders Arthur Königi), in dessen von Th. Engelmann herausgegebenen „Gesammelten Abhandlungen zur physiologischen Optik". Leipzig 1903, J. A. Barth. S. 160 .

J) Schwarz, Die Funktionsprüfung des Auges. Berlin 1904, S. Karger.

2) v. Graefe's Arch. f. Ophthalm. Bd. XXXII. S. 171 und Bd. XXXVI. 1. S. 133 .

v. Graefe's Archiv für Ophthalmologie. LIX. 1. 
Myopiegraden und zwar noch häufiger und intensiver als die Herabsetzung der Sehschärfe, und hier wie überhaupt vorwiegend als Schädigung der Reizempfindlichkeit, insbesondere der zentralen eintritt. Wenn nun auch die Schädigung des Lichtsinnes wie die der Sehschärfe in den niedrigern und mittlern Graden der Myopie eine teilweise, rorübergehende sein kann, so ist sie doch in den höhern Gradenvon 6. Dioptrien $a b$ - und in der Mehrzahl der mittlern Grade eine bleibende und bei den erstern sogar eine sehr erhebliche.

Nachdem ich nunmehr sicher nachgewiesen zu haben glaube, dass bei den Myopen der Lichtsinn noch mehr als die Sehschärfe sich geschädigt findet, glaube ich darin eine weitere Bestätigung: meiner Annahme sehen zn dürfen, dass die dem Eintritt und der Progression der Myopie zu Grunde liegende Ausbuchtung und Verdümmung der äussern Bulbuskapsel am hintern Augenpole, wie sie Schnabel nachgewiesen hat"), nicht ohne Dehnung und Zerrung der beiden innern Augenhäute, wenigstens der Sehnervenfasern und des den Sehpurpur secernierenden Neuroepithels bezw. der Stäbchen und Zapfen nebst dem Pigmentepithel vor sich geht. Wollte man, wie es von mancher Seite geschieht, die Kurzsichtigkeit als kein Gebrechen, ja sogar als einen Vorteil für viele Berufsstände ansehen, so weist uns doch die mit der Entstehung und dem Fortschreiten der Myopie einhergebende und mit ihr im Kausalaexus stehende Abnahme der Sehschärfe und Verschlechterung des Lichtsinnes, insbesondere der zentralen Reizempfindlichkeit, mit aller Entschiedenheit darauf hin, wie dringend notwendig eine energische und weitschanende Augenhygiene für die Schule ist, um wenigstens die im Vorm stehenden dargelegten nachteiligen Folgen der Myopie einzuschränken. Dass hygienische Bestrebungen von Erfolg sind, dafür glaube ich in meiner letzten in diesem Archiv erschienenen Arbeit?) den Nachweis erbracht zu haben.

2) Schnabel and Herrenheiser, ẗber Staphyloma posticum, Conus und. Myopie. Zeitschr. f. Heilk. Bd. XVI.

2) Loc. cit. 